\title{
Information Sharing in a Supply Chain with Horizontal Competition: The Case of Discount Based Incentive Scheme
}

\author{
Birendra K. Mishra ${ }^{1}$, Srinivasan Raghunathan ${ }^{2}$ \\ ${ }^{1}$ School of Management, University of California at Riverside, Riverside, USA \\ ${ }^{2}$ School of Management, University of Texas at Dallas, Richardson, USA \\ Email: barry.mishra@ucr.edu, sraghu@utdallas.edu
}

Received April 24, 2012; revised May 21, 2012; accepted June 21, 2012

\begin{abstract}
$\mathrm{Li}$ [1] examined the incentives for information sharing in a two-level supply chain in which there are a manufacturer and many competing retailers. Li showed that direct and leakage effects of information sharing discourage retailers from sharing their information and identified conditions under which demand information sharing can be traded. The purpose of this note is to show that full information is the equilibrium if the manufacturer adopts a discount based incentive scheme instead of the side-payment scheme used by Li. The discount-based scheme eliminates the direct as well as leakage effects. Discount based scheme is attractive because similar schemes are commonly used in practice and it results in Pareto-efficient information sharing equilibrium that has a higher social welfare and consumer surplus than the no information sharing scenario. The total social benefits and consumer surplus are higher in discount based incentive scheme. Consequently, many of the key results of Li are critically dependent on the assumption that the manufacturer uses side payment for information.
\end{abstract}

Keywords: Information Sharing; Supply Chain; Competition

\section{Introduction}

$\mathrm{Li}$ [1] examined the incentives for firms to share information vertically in a two-level supply chain in which there are a single upstream manufacturer and many downstream competing retailers. Li showed that while the manufacturer always benefits from retailers' demand information, retailers would not voluntarily share their information. The results is because the manufacturer, being the leader in the game, is able to exploit retailers' information to its advantage ("direct effect"), and retailers are able to infer competitors' information from manufacturer's price ("leakage effect"). However, when the manufacturer is allowed to compensate retailers for information disclosure, information sharing can be achieved when 1) the information each retailer has is relatively more informative in a statistical sense; or 2) there is sufficiently large number of retailers. $\mathrm{Li}$ also showed that complete demand information sharing reduces both the expected total social benefits and the expected consumer surplus. The purpose of this paper is to show that the key results of Li regarding the conditions when information sharing will occur, social benefits, and consumer surplus depend critically on the type as well as timing of the in- formation-sharing contract entered into by the manufacturer and retailers. Specifically, we show that if the manufacturer offers an appropriate schedule of discount on the wholesale price to retailers then full information sharing will occur under all conditions rather than only under the conditions given in $\mathrm{Li}$. In addition, consumer surplus and total social benefits increase under full information sharing in the discount-based contract.

The intuition that underlies our result becomes clear by further analysis of the reasons for the direct and leakage effects described by Li. The direct effect occurs because the manufacturer acts as the leader and sets the wholesale price based on retailers' information. When retailers' information signals a demand higher than the mean demand, the manufacturer increases the wholesale price from what it would have charged in the absence of that information. When retailers' information signals a demand smaller then the mean demand, the manufacturer charges a lower wholesale price. However, retailers lose more from a higher wholesale price in the high demand scenario than they gain from a lower wholesale price under the low demand scenario. Consequently, retailers' expected profits decrease under information sharing. In high as well as low demand scenarios the manufacturer 
benefits because it is able to set the price that maximizes its profit based on the information. This insight suggests that if an information-sharing contract is designed such that retailers do not lose in the high demand scenario and gain in the low demand scenario then it is clear that the retailers as well as the manufacturer will be better off under information sharing. One such contract is based on discounts on the wholesale price when demands are expected to be low.

The leakage effect occurs because the wholesale price reveals the signals of retailers sharing the information to other retailers. This puts those retailers that share information at a disadvantage compared to those who do not. In Li's model, the manufacturer announces the wholesale price after receiving the signals from retailers that have entered into information sharing agreement. In the contract we propose, the manufacturer announces only the discount schedule. The discount a specific retailer gets is private to the manufacturer and that retailer. This contract is consistent with industry practice ${ }^{1}$. Thus the discount based contract scheme eliminates the leakage effect of information sharing.

We briefly present the model and key results of $\mathrm{Li}$ in Section 2. We present our model and derive the principal results in Section 3. We conclude with a summary in Section 4.

\section{Li's Model and Principal Results}

Li considers a two-level supply chain with one manufacturer and $n$ retailers that sell a homogenous product. The inverse demand function for the downstream market is given by $p=a-Q$, where $p$ is the price $Q$ is the total sales level in the downstream market.

That is $Q=\sum_{i \in N} q_{i}$, where $q_{i}$ is the level of sales at retailer $i, i \in\{1,2, \ldots n\}$. The marginal cost of production is assumed to be constant and zero. The manufacturer is the Stackelberg leader and first offers a price, $P$. Then the retailers decide on their sales quantities, $q_{i}$, and the manufacturer produces the quantity $Q$. The manufacturer is obliged to meet the retailers' orders and has the capacity to do so.

$\mathrm{Li}$ analyzes two kinds of uncertainties: demand and cost. In this paper we focus only on the demand uncertainty. We can easily extend our analysis to the case of cost uncertainty and show similar results. Each retailer possesses some private information about the uncertainty. In each of these cases, the sequence of events and decisions are as follows.

${ }^{1}$ The 1936 Robinson-Patman Act precludes sellers "from giving different terms to different resellers in the same reseller class" and any proffered discount schedule must be functionally available to all retailers. Our proposed contract does not violate the act. This discount scheme is similar to quantity discount schedule widely used in practice.
1) Each retailer decides whether to disclose his information and the manufacturer decides whether to acquire such information.

2) Each retailer observes his signal and the manufacturer observes only those signals shared by the retailers.

3) Based on the available information, the manufacturer sets the wholesale price.

4) The retailers choose sales levels after receiving the wholesale price.

5) The manufacturer produces to meet the retailers' sales levels.

In demand uncertainty, the downstream demand curve is assumed to be $p=a+\theta-Q$, where $\theta$ is a random variable with zero mean variance $\sigma^{2} \equiv \operatorname{Var}[\theta]$. Before making the quantity decision, each retailer $i$ observes a signal $Y_{i}$ about $\theta$. The following assumptions were made about $Y_{i}$.

Assumption 1: $E\left[Y_{i} \mid \theta\right]=\theta$ for all $i$.

Assumption 2: $E\left[\theta \mid Y_{1}, Y_{2}, \ldots, Y_{n}\right]=\alpha_{0}+\sum_{i \in N} \alpha_{i} Y_{i}$, where $\alpha_{i}$ are constants. $Y_{i}$ are independent, conditional on $\theta$.

Assumption 3: $Y_{1}, Y_{2}, \cdots, Y_{n}$ are identically distributed.

The joint probability distribution for $\left(\theta, Y_{1}, Y_{2}, \ldots, Y_{n}\right)$ is common knowledge.

Under the above assumptions, the following holds when $k$ retailers share their information with the manufacturer.

$$
E\left[\theta \mid Y_{j}, j \in K\right]=E\left[Y_{i} \mid Y_{j}\right]=\frac{1}{k+s} \sum_{j \in K} Y_{j}
$$

where

$$
s \equiv \frac{E\left[\operatorname{Var}\left[Y_{i} \mid \theta\right]\right]}{\operatorname{Var}[\theta]}
$$

Li derives the following optimal quantities, wholesale price, and profits when $k$ retailers share their information with the manufacturer.

$$
\begin{gathered}
p^{*}\left(\left(Y_{j}\right)_{j \in K}\right)=\frac{a}{2}+\frac{A_{1}^{k}}{2} \sum_{j \in K} Y_{j} \\
q_{i}^{*}\left(Y_{i}, P^{*}\left(\left(Y_{j}\right)_{j \in K}\right)\right) \\
=\frac{1}{n+1}\left(a-P+A_{1}^{k} \sum_{j \in K} Y_{j}\right) \\
=\frac{1}{n+1}\left(\frac{a}{2}+\frac{A_{1}^{k}}{2} \sum_{j \in K} Y_{j}\right) \text { for } i \in K \\
q_{i}^{*}\left(Y_{i}, P^{*}\left(\left(Y_{j}\right)_{j \in K}\right)\right)=\frac{1}{n+1}\left(a-P+B_{1}^{k} \sum_{j \in K} Y_{j}+B_{2}^{k} Y_{i}\right)
\end{gathered}
$$




$$
\begin{gathered}
=\frac{1}{n+1}\left(\frac{a}{2}+\left(B_{1}^{k}-\frac{A_{1}^{k}}{2}\right) \sum_{j \in K} Y_{j}+B_{2}^{k} Y_{i}\right) \text { for } i \in N / K \\
\pi_{i}(k) \equiv E\left[\left(q_{i}^{*}\right)^{2}\right] \\
\pi_{\mathrm{M}}(k) \equiv \frac{n}{n+1} E\left[\left(P^{*}\right)^{2}\right]
\end{gathered}
$$

where

$$
\begin{gathered}
A_{1}^{k} \equiv \frac{1}{k+s} \\
B_{1}^{k} \equiv \frac{k+2 s}{(k+s)(n+k+1+2 s)} \\
B_{2}^{k} \equiv \frac{n+1}{n+k+1+2 s}
\end{gathered}
$$

Using the above expressions, Li shows in Proposition 4 that the manufacturer is better off by acquiring information from more retailers, and each retailer is worse off by disclosing his information to the manufacturer in all circumstances. Therefore, no information sharing is the unique equilibrium. Li then proceeds to analyze whether information sharing can be achieved when the manufacturer is allowed to compensate retailers for information disclosure. Li considers the following contract signing game in the first stage. In the contract, the manufacturer offers a payment $\delta$ to each retailer's private information. All retailers simultaneously decide whether to sign the contract. Under this contract, Li shows in Proposition 5 that there exists a $\delta$ such that complete information sharing equilibrium Pareto dominates no information sharing equilibrium if and only if $s \leq(n-2)(n+1) / 2$. That is, information sharing equilibrium Pareto dominates the no information sharing equilibrium only when $s$ is sufficiently small and/or $n$ is very large. When $n=2$, information sharing equilibrium does not Pareto dominate no information sharing equilibrium. In Proposition 7, Li shows that complete information sharing reduces both the expected total social benefits and the expected consumer surplus given by $(a+\theta) E[Q]-E\left[Q^{2}\right] / 2$ and $E\left[Q^{2}\right] / 2$ respectively, where

$$
Q=\sum_{i \in K} q_{i}^{*}+\sum_{i \in N / K} q_{i}^{*}
$$

\section{Our Model and Analysis}

\subsection{Our Model}

It is worth noting that the contract of $\mathrm{Li}$ is based on a fixed payment of $\delta$ and not on the wholesale price. However, it is well known that the profits of the manufacturer, retailers, and the overall supply chain depend critically on the wholesale price because of the double marginalization effect [2]. In a deterministic demand situation, a higher (lower) wholesale price increases (decreases) the manufacturer profit but reduces (increases) retailers' and the supply chain's profits. Li shows the intuitive result that information sharing will occur only when the supply chain profit increases as a result of information sharing. When the manufacturer sets the wholesale price first to maximize its own profit, the supply chain profit improves from information sharing only under certain conditions. When these conditions are satisfied, the manufacturer can indeed use the contract proposed by $\mathrm{Li}$ and realize higher profits. However, when the conditions are not satisfied, information sharing is not achieved under the side payment contract. We show in the following paragraphs that if the manufacturer uses a contract based on the wholesale price then information sharing equilibrium can be achieved, and the manufacturer as well as retailers benefit as well.

The intuition for the contract we propose is based on a simple proposition ${ }^{2}$. If the manufacturer and retailers enter into a contract such that neither the retailer nor the manufacturer is worse off when information is shared compared to when information is not shared under all realizations of the random signals observed by the retailer then information sharing equilibrium will Pareto dominate the no information equilibrium under all circumstances. For any set of realizations of the signals, a higher wholesale price under information sharing benefits the manufacturer and hurts retailers. Consequently if the manufacturer agrees to not increase the wholesale price from what he would have charged under no information sharing, retailers will not be worse off. As for the manufacturer, if the manufacturer deems that it will benefit from giving a discount after the information is shared, it will benefit by offering the discount. If the manufacturer neither gives a discount nor raises its price based on information shared by retailers, the manufacturer will not be worse off compared to the no information sharing scenario. Such a contract results in a win-win situation for both manufacturer and retailers. We formally state our wholesale price scheme based on discounts as follows.

Discount scheme: $P=a / 2-D Y$ where $D \leq 0$ is the discount rate if the shared signal is $Y \leq 0$, and $D$ is equal to 0 if $Y$ is not shared or $Y>0$.

We show that there exists a discount rate $D$ such that when the manufacturer offers this discount schedule all retailers will share information and that both retailers and

\footnotetext{
${ }^{2}$ It should be emphasized that the contract we propose is not the only possible wholesale price based contract to achieve information sharing equilibrium. Also, several other contracts based on wholesale price as well as side payments exist that can achieve this equilibrium. Our choice of the wholesale priced contract is based on the fact the proposed contract is simple to implement and captures discounts, a commonly employed method to "buy" retailer information.
} 
the manufacturer in the information sharing equilibrium than the no information sharing equilibrium. We also use the following sequence of actions in our analysis in order to make the above schedule available to all retailers prior to their making the decision on whether to share information.

1) The manufacturer offers the discount price schedule.

2) Each retailer decides whether to disclose his information and the manufacturer decides whether to acquire such information.

3) Each retailer observes his signal and the manufacturer observes only those signals shared by the retailers.

4) Based on the discount price schedule, the manufacturer offers the discount to those retailers that shared the information.

5) The retailers choose sales levels after receiving the wholesale price.

6) The manufacturer produces to meet the retailers' sales levels.

The rest of the model remains the same as that of $\mathrm{Li}$.

\subsection{Analysis of Our Discount Based Price Scheme}

We first derive the optimal sales quantities when $k \geq 0$ retailers share their information with the manufacturer. In the last stage of the game, the expected profit for retailer $i$, given his information, is

$$
\begin{aligned}
& E\left[\pi_{i} \mid Y_{i}\right] \\
& =\left(a+E\left[\theta \mid Y_{i}\right]-q_{i}-\sum_{l \neq i} E\left[q_{l} \mid Y_{i}\right]-\frac{a}{2}+D Y_{i}\right) q_{i} .
\end{aligned}
$$

The equilibrium sales quantity must satisfy the firstorder condition:

$$
2 q_{i}^{*}=\frac{a}{2}+D Y_{i}+E\left[\theta \mid Y_{i}\right]-E\left[\sum_{l \neq i} q_{1}^{*} \mid Y_{i}\right] .
$$

As in Li, we use Bayesian Nash equilibrium to derive the optimal sales levels. A Bayesian Nash equilibrium is a set of strategies and a set of conjectures such that 1) each firm strategy is a best response to its conjecture about the behaviors of its rivals; and 2) the conjectures are correct [3]. We assume that each retailer conjectures that each of the other retailers' sales quantity is a linear function of its own signal, and we shall show that this conjecture is correct in equilibrium. That is, let

$$
q_{i}^{*}=\alpha+\beta Y_{i} .
$$

Then, Equation (12) becomes

$$
\begin{aligned}
2 \alpha+2 \beta Y_{i} & =\frac{a}{2}+D Y_{i}+E\left[\theta \mid Y_{i}\right]-(n-1) \alpha-\beta E\left[\sum_{l \neq i} Y_{l} \mid Y_{i}\right] \\
& =\frac{a}{2}+D Y_{i}+A_{1}^{0} Y_{i}-(n-1) \alpha-\beta(n-1) A_{1}^{0} Y_{i} .
\end{aligned}
$$

Thus, we get

$$
\begin{aligned}
& 2 \alpha=\frac{a}{2}-(n-1) \alpha \Rightarrow \alpha=\frac{a}{2(n+1)} \\
& 2 \beta Y_{i}=D Y_{i}+A_{1}^{0} Y_{i}-\beta(n-1) A_{1}^{0} Y_{i} \\
& \Rightarrow \beta=\frac{D+A_{1}^{0}}{2+(n-1) A_{1}^{0}}=\frac{1+D(1+s)}{n+2 s+1} .
\end{aligned}
$$

Note that our conjecture that the sales quantity for any retailer is a linear function of its own signal is correct in the equilibrium. The equilibrium sales quantity for retailer $i$ is then given by

$$
q_{i}^{*}=\frac{1}{n+1}\left(\frac{a}{2}+B_{2}^{0}(1+D(1+s)) Y_{i}\right)
$$

The manufacturer's expected profit in the preceding stage given her information $\left(Y_{j}\right)_{j \in K}$ is given by

$$
\begin{aligned}
& E\left[\pi_{M} \mid\left(Y_{j}\right)_{j \in K}\right] \\
& =\sum_{i \in N} E\left[\left(\frac{a}{2}-D Y_{i}\right) q_{i}^{*} \mid\left(Y_{j}\right)_{j \in K}\right] \\
& =\sum_{i \in N} \frac{1}{n+1} E\left[\left(\frac{a}{2}-D Y_{i}\right)\left(\frac{a}{2}+B_{2}^{0}(1+D(1+s)) Y_{i}\right) \mid\left(Y_{j}\right)_{j \in K}\right] .
\end{aligned}
$$

Note that the equilibrium wholesale price and sales quantity for a retailer are dependent only on that retailer's information, and whether the information is shared with the manufacturer. Specifically, they do not depend on how many retailers share their information. Consequently, leakage effect from information sharing is eliminated.

The retailer profits can now be computed as

$$
\begin{aligned}
\pi_{i} & =E\left[\left(q_{i}^{*}\right)^{2}\right] \\
& =\frac{1}{4(n+1)^{2}}\left(\int_{-A}^{0}\left(a+B_{2}^{0}(1+D(1+s)) Y_{i}\right)^{2} f(z) \mathrm{d} z\right), i \in K \\
& =\frac{1}{4(n+1)^{2}}\left(\int_{0}^{B}\left(a+2 B_{2}^{0} Y_{i}\right)^{2} f(z) \mathrm{d} z\right. \\
& =\frac{a^{2}}{4(n+1)^{2}}+\frac{\sigma^{2}}{(n+1)^{2}}\left(B_{2}^{0}\right)^{2}(1+s), i \in N / K .
\end{aligned}
$$

Now, we can show the following result about the number of retailers that will share information in the equilibrium under our discount price schedule.

Proposition 1: For any $D \leq 0$, full information sharing in which all retailers share their information is the unique equilibrium.

Proof: The proof is straightforward. It follows from the fact that irrespective of the number of retailers that 
already share information, a retailer that does not share his information can earn higher profit by sharing its information. That is,

$$
\begin{aligned}
& \frac{1}{4(n+1)^{2}}\left(\begin{array}{l}
\left.\int_{-A}^{0}\left(a+B_{2}^{0}(1+D(1+s)) Y_{i}\right)^{2} f(z) \mathrm{d} z\right) \\
+\int_{0}^{B}\left(a+2 B_{2}^{0} Y_{i}\right)^{2} f(z) \mathrm{d} z
\end{array}\right) \\
& \geq \frac{1}{4(n+1)^{2}}\left(\int_{-A}^{B}\left(a+2 B_{2}^{0} Y_{i}\right)^{2} f(z) \mathrm{d} z\right) .
\end{aligned}
$$

The following result shows that the manufacturer as well as retailers prefers the full information sharing scenario compared to the no information sharing scenario.

Proposition 2: There exists a $D$ such that both the manufacturer and retailers are better off under full information sharing than under no information sharing.

Proof: The proof for Proposition 1 shows that the profits of retailers are higher under the full information scenario than no information scenario. We can easily show that for $Y_{i}<0,\left(a^{2}-\left(B_{2}^{0} Y_{i}\right)^{2}\right) \geq a\left(a+2 B_{2}^{0} Y_{i}\right)$. Consequently, the manufacturer' profit is higher under full information sharing scenario. Q.E.D.

Having shown that full information sharing in which both the manufacturer and retailers are better off is the equilibrium under our discount price based schedule, an interesting question for the manufacturer is which type of contract, discount based or fixed payment as in $\mathrm{Li}$, will the manufacturer prefer. Under the contract analyzed in $\mathrm{Li}$, the manufacturer realizes an additional profit of

$$
\frac{\sigma^{2} n^{2}((n+1)(n-1)-s)}{4(n+1)^{2}(n-1+s)(n+s)},
$$

under information sharing ([2], p. 1204). Note that this additional profit is non-negative if and only if $s \leq(n-2)(n+1) / 2$. In our model, the additional profit is always non-negative and is given by

$$
\frac{n}{4(n+1)}\left(\int_{-A}^{0}-\left(B_{2}^{0} Y_{i}\right)^{2} f(z) \mathrm{d} z+\int_{0}^{B} 2 a B_{2}^{0} Y_{i} f(z) \mathrm{d} z\right) .
$$

The manufacturer will prefer the discount-based contract when either of the following two conditions are satisfied.

1) $s>(n-2)(n+1) / 2$

2) $a>\frac{\frac{\sigma^{2} n((n+1)(n-1)-s)}{(n+1)(n-1+s)(n+s)}+\left(B_{2}^{0}\right)^{2} \int_{-A}^{0}\left(Y_{i}\right)^{2} f(z) d z}{2 B_{2}^{0} \int_{0}^{B} Y_{i} f(z) d z}$

Observe that under condition 1), information is not shared in Li's model. However, under our discount scheme both the manufacturer and retailers benefit from information sharing. Condition 2) follows a comparison of the manufacturer's benefits from information sharing under Li's side payment mechanism

$$
\text { (i.e., } \left.\frac{\sigma^{2} n^{2}((n+1)(n-1)-s)}{4(n+1)^{2}(n-1+s)(n+s)}\right)
$$

and our discount scheme

$$
\text { (i.e., } \frac{n}{4(n+1)}\left(\int_{-A}^{0}-\left(B_{2}^{0} Y_{i}\right)^{2} f(z) \mathrm{d} z+\int_{0}^{B} 2 a B_{2}^{0} Y_{i} f(z) \mathrm{d} z\right) \text { ). }
$$

The conditions imply that when the number of retailers is small and/or the signal accuracy is large so that fixed payment based contract is unprofitable or when the mean demand is sufficiently large, the manufacturer should use discount on the wholesale price to induce retailers to share information sharing.

Finally, we also analyze the effect of discount-based scheme on the social welfare and consumer surplus. The consumer surplus CS under our discount scheme is given by

$$
\begin{aligned}
& C S=\frac{1}{2} E\left[Q^{2}\right] \\
& =\frac{n}{8(n+1)^{2}}\left(\int_{-A}^{0}\left(a+B_{2}^{0} Y_{i}\right)^{2} f(z) \mathrm{d} z+\int_{0}^{B}\left(a+2 B_{2}^{0} Y_{i}\right)^{2} f(z) \mathrm{d} z\right)
\end{aligned}
$$

The social welfare is given by $n \pi_{i}+\pi_{M}+C S$.

Proposition 3: Under the discount scheme, the consumer surplus and social welfare are higher under full information sharing than no information sharing.

Proof: The consumer surplus under full information sharing is given by Equation (16). Consumer surplus under no information sharing is given by

$$
\frac{n}{8(n+1)^{2}}\left(\int_{-A}^{B}\left(a+2 B_{2}^{0} Y_{i}\right)^{2} f(z) d z\right) .
$$

Since $\int_{-A}^{0}\left(a+B_{2}^{0} Y_{i}\right)^{2} f(z) \mathrm{d} z>\int_{-A}^{0}\left(a+2 B_{2}^{0} Y_{i}\right)^{2} f(z) \mathrm{d} z$, it follows that consumer surplus is higher under information sharing. Q.E.D.

The result that social welfare is higher under information sharing follows from the results that the manufacturer profit, retailers' profit, as well as consumer surplus are higher under information sharing.

\section{Discussion}

We showed in this paper that many of the key results of $\mathrm{Li}$, especially those related to the conditions under which vertical demand information sharing will occur in a supply chain with horizontal competition and the effect of demand information sharing on consumer surplus and 
social welfare, are critically dependent on the assumption that the manufacturer uses side payment for (or "buys") information. In this setup, the manufacturer retains the right to set any wholesale price based on available information. The wholesale price also signals the information to those that do not share information. It is well known that retailer profits as well as total supply chain profit depend critically on the wholesale price. A higher wholesale price reduces retailer profits and supply chain profit. Consequently, in Li's set up, under certain conditions, the payment the manufacturer should make to induce retailers to share information becomes so high that it is unprofitable for the manufacturer. We proposed an alternative contract based on wholesale price discount scheme to induce information sharing. The scheme is attractive for a variety of reasons. First, wholesale price discount based scheme is commonly used in practice in information sharing settings $[4]^{3}$. Second, the discount scheme results in Pareto-optimal information sharing equilibrium for all supply chain structures and demand conditions. Third, the scheme also increases the consumer surplus and social welfare. We also analyzed when a manufacturer that is a Stackelberg leader prefers the discount based scheme vis-à-vis the side payment scheme.

The paper can be extended in several directions. We considered a specific wholesale price based contract in which the manufacturer commits, under information sharing, not to increase the wholesale price from that under no information sharing. A worthwhile problem to investigate is whether there are other contracts that yield simi- lar results. We believe that numerous contracts that use a combination of discount and side payment can be designed that will yield qualitatively similar results. However, depending on the contract, the manufacturer's and retailers' shares of the supply chain profit will vary. In our setup as well Li's, only retailers get signals about the demand. We could allow the manufacturer to obtain signals in addition to the retailers. If the manufacturer's signal is significantly more accurate than those of retailers, information sharing may become less attractive from the manufacturer's perspective. Another direction for further research is to allow the signals - both retailers' as well as manufacturer's - to be correlated. Higher correlation will make information sharing less valuable.

\section{REFERENCES}

[1] L. Li, "Information Sharing in a Supply Chain with Horizontal Competition," Management Science, Vol. 48, No. 9, 2002, pp. 1196-1212. doi:10.1287/mnsc.48.9.1196.177.

[2] J. Spengler, "Vertical Integration and Antitrust Policy," Journal of Political Economy, Vol. 58, No. 4, 1950, pp. 347-352. doi:10.1086/256964

[3] J. Harsanyi, "Bayesian Decision Theory and Utilitarian Ethics," The American Economic Review, Vol. 68, No. 2, 1978, pp. 223-228.

[4] H. Lee, K. So and C. Tang, "The Value of Information Sharing in a Two-Level Supply Chain," Management Science, Vol. 46, No. 5, 2000, pp. 626-643. doi:10.1287/mnsc.46.5.626.12047

\footnotetext{
${ }^{3}$ Other incentive schemes reported by Lee, So, and Tang are better return policy, better payment terms, reduction in replenishment lead time, and Vendor Managed Inventory (VMI) (Page 638).
} 